\title{
Correspondence Critical Issues in Cross-sectional Research Studies of Human Ecstasy (MDMA) Users: A Response to Krebs and Johansen
}

\author{
Ronald L Cowan*,I \\ 'Psychiatric Neuroimaging Program, Vanderbilt Addiction Center, Vanderbilt University School of Medicine, Nashville, TN, USA
}

Neuropsychopharmacology (2012) 37, 1072-1073; doi: I0.1038/npp.201 I.223

In a letter to the editor entitled 'Methodological weaknesses in non-randomized studies of ecstasy (MDMA) use: a cautionary note to readers and reviewers', Krebs and Johansen comment on our recent manuscript, 'Human ecstasy use is associated with increased cortical excitability: an fMRI study' (Bauernfeind et al, 2011) and highlight important concerns inherent in the conduct of cross-sectional research in human ecstasy (MDMA) users. We are in considerable agreement with the authors regarding the need for well-controlled longitudinal studies to more precisely address the potential neurotoxic effects of recreational MDMA exposure. Although the concerns raised by Krebs and Johansen were addressed in our original report, several points merit additional consideration and clarification.

In addition to commenting on our peer-reviewed manuscript (Bauernfeind et al, 2011), Krebs and Johansen note that the principal investigator (RLC) compares MDMA users to people with early Alzheimer's disease (AD) in a press release. In prior peer-reviewed publications (Karageorgiou et al, 2009; Cowan et al, 2006), we have noted the overlap between findings of increased task-evoked activation in MDMA users and in individuals at risk for AD. These increased task or stimulus-evoked activations may be a marker for reduced neural efficiency and a compensatory response to inefficient brain function. Thus, MDMA users and those with risk for AD show overlap with regard to their blood oxygenation level dependent (BOLD) signal characteristics. We neither posit that the primary cause of the increased BOLD signal is similar in MDMA users and people with $A D$ nor do we suggest that MDMA users will develop $\mathrm{AD}$ or other dementias at increased rates. However, to the degree that increased BOLD signal may reflect increased cortical excitability in both people with $\mathrm{AD}$ and in MDMA users - possibly due to shifts in excitatory or inhibitory neurotransmission - the secondary effects of such a shift in cortical physiology may be similar.

*Correspondence: Dr RL Cowan, Psychiatric Neuroimaging Program, Vanderbilt Addiction Center, Vanderbilt University School of Medicine, I60I 23rd Avenue South, Suite 3057, Nashville, TN 37212, USA, Tel: 615322 2303, E-mail: ronald.l.cowan@vanderbilt.edu

Received 16 August 2011; accepted 19 August 2011
Krebs and Johansen reanalyzed our raw data by combining MDMA user and control data into a single correlation analysis, and report that they found no evidence for an association of MDMA exposure and brain activation. As we discussed in our original manuscript (Bauernfeind et al, 2011), and in prior work in different cohorts, low-dose MDMA use tends to be associated with activation values that are somewhat lower than the control cohort mean whereas high-dose MDMA use tends to be associated with activation values greater than the control cohort mean. As we wanted to begin to address the question of the effect of low-dose MDMA on brain function, our study enrolled individuals having between 3 and 155 episodes of MDMA use and, therefore, included individuals with very-low MDMA exposure levels. A simple average of the means or a correlation analysis that includes the control cohort, as performed by Krebs and Johansen (when the MDMA cohort includes very-low-dose MDMA users), therefore, inappropriately obscures the strong dose-response relationship between lifetime MDMA exposure and brain activation. This relationship is evident in the manuscript and in the associated correlation analyses when ranked data is used to properly account for the non-parametric distribution of lifetime MDMA use (Bauernfeind et al, 2011).

Krebs and Johansen point out the possibility that psychological factors associated with cortical activation (eg, impulsivity) may predispose one to MDMA use. Although we agree with this possibility, MDMA has a well-established ability to cause serotonin neurotoxicity in non-human primates, and we are not aware of evidence to suggest that humans are less vulnerable to these effects. We have also recently shown that MDMA use is associated with alterations in functional connectivity (Salomon et al, 2011) that are consistent with reduced serotonin function. Irrespective of pre-existing differences, we believe it is likely that MDMAinduced serotonergic neurotoxicity leads to changes in mood and behavior. For example, in a prospective study of MDMA users, de Winn et al, 2007 found that incident MDMA use was associated with an increase in impulsivity. These findings suggest that MDMA use may lead to greater impulsivity via changes in serotonin function and that elevated impulsivity is not solely a pre-existing trait. 
Overall, Krebs and Johansen raise important theoretical concerns regarding our report and similar reports in the human MDMA literature. To confirm or refute findings from cross-sectional studies, large well-controlled prospective studies of the effects of MDMA use on brain function in humans are greatly needed. However, much can be learned from well-designed cross-sectional neuroimaging studies that incorporate an understanding of serotonin neurophysiology and of the neural consequences of MDMA exposure that have been demonstrated extensively in animals (Cowan et al, 2008; Reneman et al, 2006).

\section{ACKNOWLEDGEMENTS}

This work was supported by NIDA/NIH (R01 DA01537 and R21 DA020149 to RLC, and NCRR/NIH (Vanderbilt CTSA Grant UL1 RR024975).

\section{DISCLOSURE}

RLC received publication royalties from Lippincott Williams and Wilkins, and consultant income from the Southwest Michigan First Life Science Fund, the University of West Alabama, Novo Nordisk, the Treatment Research Institute and Shire Pharmaceuticals.

\section{REFERENCES}

Bauernfeind A, Dietrich M, Blackford J, Charboneau E, Lillevig J, Cannistraci C et al (2011). Human Ecstasy use is associated with increased cortical excitability: an fMRI study. Neuropsychopharmacology 36: 1127-1141.

Cowan RL, Haga E, deB FB, Dietrich MS, Vimal RL, Lukas SE et al (2006). MDMA use is associated with increased spatial BOLD fMRI visual cortex activation in human MDMA users. Pharmacol Biochem Behav 84: 219-228.

Cowan RL, Roberts DM, Joers JM (2008). Neuroimaging in human MDMA (Ecstasy) users. Ann N Y Acad Sci 1139: 291-298.

de Win MM, Reneman L, Jager G, Vlieger EJ, Olabarriaga SD, Lavini C et al (2007). A prospective cohort study on sustained effects of low-dose ecstasy use on the brain in new ecstasy users. Neuropsychopharmacology 32: 458-470.

Karageorgiou J, Dietrich MS, Charboneau EJ, Woodward ND, Blackford JU, Salomon RM et al (2009). Prior MDMA (Ecstasy) use is associated with increased basal ganglia-thalamocortical circuit activation during motor task performance in humans: an fMRI study. Neuroimage 46: 817-826.

Reneman L, de Win MM, van den Brink W, Booij J, den Heeten GJ (2006). Neuroimaging findings with MDMA/ecstasy: technical aspects, conceptual issues and future prospects. J Psychopharmacol 20: 164-175.

Salomon RM, Karageorgiou J, Dietrich MS, McLellan JY, Charboneau EJ, Blackford JU et al (2011). MDMA (Ecstasy) association with impaired fMRI BOLD thalamic coherence and functional connectivity. Drug Alcohol Depend (e-pub ahead of print 30 July 2011). 